\title{
Fetal Brain Vascularity Visualized by Conventional 2D and 3D Power Doppler Technology
}

\author{
${ }^{1}$ Ritsuko K Pooh, ${ }^{2}$ Asim Kurjak \\ ${ }^{1}$ CRIFM Clinical Research Institute of Fetal Medicine PMC, Osaka, Japan \\ ${ }^{2}$ Department of Obstetrics and Gynecology, University of Zagreb, School of Medicine, Zagreb, Croatia
}

Correspondence: Ritsuko K Pooh, CRIFM Clinical Research Institute of Fetal Medicine PMC 7-3-7, Uehommachi, Tennoji Osaka \#543-0001, Japan, Phone: +81-6-6775-8111, Fax: +81-6-6775-8122, e-mail: rkpooh@me.com

\begin{abstract}
Significant advances have been made in accurate and reliable visualization of the cerebral circulation in normal and abnormal pregnancies. They provided the non-invasive studies of fetal cerebral angiogenesis and further development that filled some of the gaps made by neuroanatomical studies alone. The first breakthrough in the assessment of fetal circulation was development of Doppler system with purpose to obtain velocity waveforms. Continuing technical advances in Doppler ultrasound equipment, especially highly sensitive color flow imagining techniques have made it possible to study smaller anatomical parts of fetal circulation system including cerebral vascularization. Before examination of brain vascularity, anatomical vascular structure and development on the different appearance at each gestational age should be remembered as the basic knowledge. Since the development of the embryo is rapid and significant changes occur during even one week it is important to specify the stage of the embryo or fetus both by age (postmenstrual weeks and days) and by size (crownrump length (CRL) and biparietal diameter (BPD).

Introduction of three-dimensional (3D) sonography and 3D power Doppler techniques have enabled visualization of intracranial vessels. 3D power Doppler assessment particularly adds useful information in the prenatal evaluation of normal brain development, vascular malformation and tumoral vascularity.
\end{abstract}

Keywords: Early fetal circulation, Cerebral circulation, 2D color Doppler, 3D sonography, 3D power Doppler, Angiogenesis.

\section{VASCULARITY VISUALIZED BY CONVENTIONAL 2D AND 3D COLOR/POWER DOPPLER TECHNOLOGY}

Early fetal circulation has been demonstrated by conventional 2D color Doppler from 1990s. ${ }^{1}$ Fine vessels inside of choroid plexus were depicted and assessed in 1994 . $^{2,3}$ 2D color/power Doppler combined with transvaginal sonography became a powerful tool to demonstrate early fetal vascularization. ${ }^{4}$ Sonoangiograms shown in Figures 1 and 2 were taken in 1996 by authors. The internal carotid arteries, anterior/middle cerebral arteries are well-demonstrated in the first trimester. With advance of power Doppler technology, 2D power Doppler had depicted more precisely the intracerebral vascularity as shown in Figure 3. The first venous Doppler study was reported in 1999 by Pooh et al. ${ }^{5}$ As shown in Figure 4, 2D color Doppler image depicts the dural sinuses, superior sagittal sinus, internal cerebral vein, vein of Galen, and straight sinus, with normal palsatile blood flow waveform pattern. ${ }^{5}$ 3D technology had advanced in late 1990s and 3D power Doppler combined with transvaginal sonography have been able to provide threedimensional sonoangiographic images from $2000^{4}$ as shown in Figure 5.

\section{MORPHOLOGICAL DEVELOPMENT OF CEREBRAL CIRCULATION}

Angiogenesis is a process of sprouting the capillaries necessary for every fetal organ to develop. Intraneural vascularization is regulated by angiogenesis factor which, at a certain time of brain development, stimulate the proliferation of endothelial cells and chemotatically attract the capillaries. Biologist Risau in 1986 has shown that it is possible to isolate angiogenesis factors from embryonic

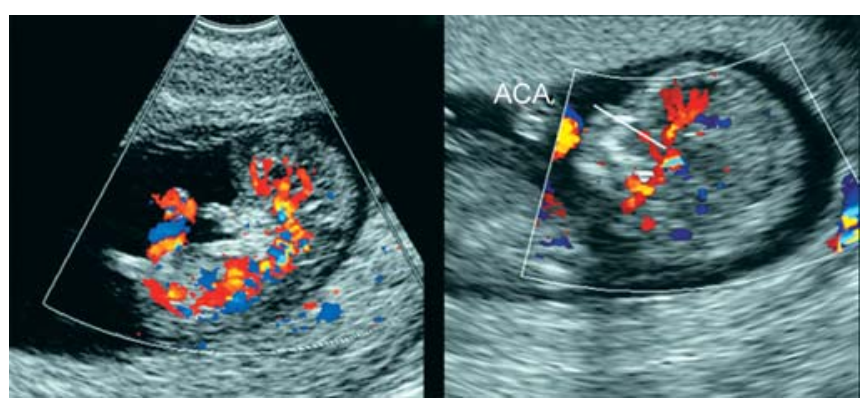

Fig. 1: Early cerebral vascularity by conventional color Doppler. (left) The sagittal sonographic embryonal image at the beginning of 10th week. Premature cerebral vascular structure is shown. (right) The sagittal image of cerebral vascularity at 12 th week. Anteriorcerebral artery (ACA) and its branches are well-demonstrated 




Fig. 2: Intracerebral circulation by conventional power Doppler in early pregnancy.Coronal image at 13th week. Bilateral internal carotid arteries (ICA) and middle cerebral arteries (MCA) are demonstrated

tissue as small as a 3-day chicken brain. This investigation supported the view that intraneural vessels do not originate in situ but invade the neural tissue from the perineural vascular system stimulated by angiogenesis factors that are present early in embryonic development. ${ }^{6}$ The most important cells for vasculogenesis are endothelial cells deriving from mesoderm with great functional heterogeneity in different organs and tissues under physiological as well as pathological conditions. Vascular endothelial cells cover the entire inner surface of blood vessels and are influenced by vascular endothelial growth factor (VEGF) which is a potent angiogenic factor working as an endothelial cellspecific mitogen and exerting atrophic effect on neurons and glial cells, both these activities being essential during central nervous system vascularization, development and repair. In these cells VEGF expression appears develop- mentally regulated and is correlated with angiogenesis, which in turn, responds to the high metabolic demands of the developing fetal brain. ${ }^{7}$

Approximately at the beginning of third week of gestation the cells of visceral mesoderm in the wall of yolk sac differentiate into blood and endothelial cells. At first there are angioblasts that form isolated angiogenic masses with cavities between cells that joined and form lumen of vessels. The cells that stayed at the center of these cavities became primitive blood cells while those from the periphery became flat forming the endothelia. These transformed angiogenic masses are closing to each other (influenced by previously mentioned factors) forming first true blood vessels.

From the beginning of cardiac formation cranial parts of truncus arteriosus enlarged into aortic sac those later form aortic branches. After the splitting of truncus arteriosus with aorticopulmonal septum aortic sac became the part of ventral aorta, while a pair of dorsal aorta raise independently of cardiac tube. In later development great changes occur leading to the lost of symmetrical state of these primitive vessels. In brief, the common aortic artery along with the first part of internal carotid artery arises from the third aortic branch, while the rest of the vessel develops from cranial part of dorsal aorta. External carotid artery develops as a cranial part of third aortic branch, while vertebral arteries merge from dorsal aorta. In the development of cerebral fetal veins the most important role has upper cardinal veins that drain the blood from cranial part of embryo.

During fetal development (approximately 35 gestational days) the craniocerebral circulation is characterized by temporary connections between the primitive carotid and the paired dorsal longitudinal neural arteries (precursors of the vertebrobasilar system) appear. They include persistent



Fig. 3: Brain circulation by conventional 2D power Doppler. (left) Axial image. Circle of Willis (W) and middle cerebral arteries (MCA) are demonstrated. (middle) Coronal image. Internal carotid arteries (ICA) and branches of middle cerebral arteries are detected. (right) Sagittal image. Anterior cerebral artery (ACA), pericallosal artery (PCA) and branches are demonstrated 


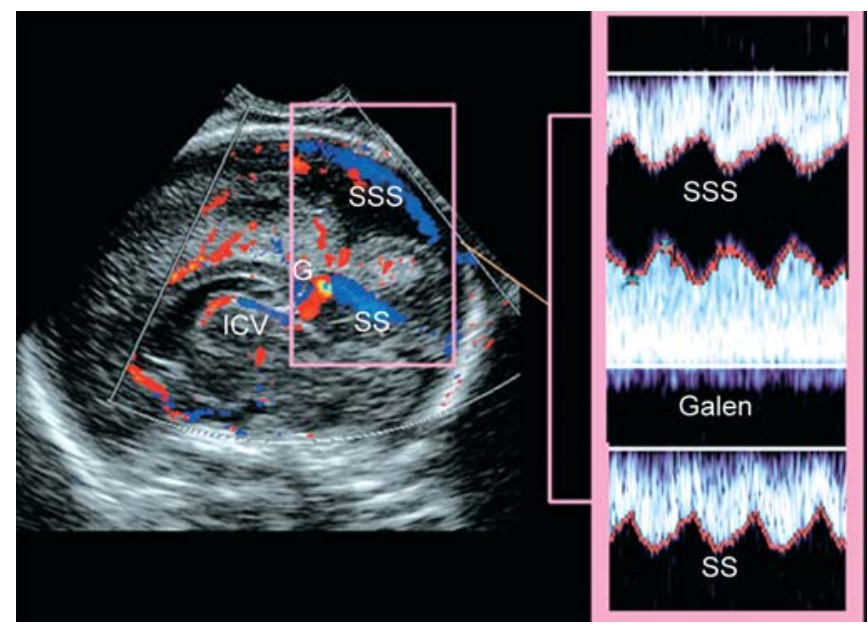

Fig. 4: Normal cerebral venous circulation by conventional 2D color Doppler. (left) Sagittal image of color Doppler. SSS—superior sagittal sinus; ICV—internal cerebral vein; G—vein of Galen; SS—straight sinus (right); Normal blood flow waveforms of dural sinuses

trigeminal, ottic, hypoglossal and proatlantic intersegmental arteries. Normal embryonic development underwrites the regression of all these vessels totally. Sometimes the regression does not occur resulting with persistent trigeminal artery which is the most frequent (85\%) of this primitive carotid-basilar anastomoses. ${ }^{8}$

\section{FETAL CNS VASCULARIZATION BY RECENT ADVANCED 2D/3D DOPPLER WITH HIGH-FREQUENCY TRANSVAGINAL SONOGRAPHY}

First assessment of cerebral vascularization is possible from 7th week of gestation with low velocity signals and absence of the diastolic flow from the periphery of the rhombencephalic cavity. ${ }^{3}$ At that stage the lateral ventricles are small evaginations located lateral and rostral from the cavity of diencephalons which continuous directly into that of the mesencephalon (Fig. 6). The isthmus rhombencephali represents the connection to the rombencephalic cavity and future fourth ventricle. The cavity of rhombencephalon is largest brain cavity visible in the embryonic head as shown in Figure 6. From 8 weeks of gestation, it is possible to detect blood flow signals from internal carotid and vertebral arteries. At this stage, rhombencephalic cavity (future fourth ventricle) decreases its size and moves towards occipital part. Upon reaching the brain, two internal carotid arteries turn dorsally and alongside the diencephalons giving off branches (Fig. 7). The internal carotid artery and its branches; anterior cerebral artery, middle cerebral artery and posterior communicating artery, form blood flow called circle of Willis that mainly supply the blood flow to cortical areas. Other vessels that form the circle are vertebral arteries running between transverse processes of C2 to C6 and giving branch called basilar artery and then posterior cerebral artery. The main components of the circulus arteriosus are present at carnegie stage 16 and the circle is complete with carnegie stage 16. Arteries originating from the vertebral arteries are important for supply of the cerebellum and brainstem. Within the symmetrical cerebellar hemispheres it is possible to visualize intracerebellar arteries. Blood flow signals from the intracerebellar arteries can be obtained from the 9 weeks of gestation as shown in Figure 8. After 9th week, cerebellar superior, anterior inferior and posterior inferior can be detected and discriminated from other cerebral arteries. They are characterized by low-to-moderate impedance to blood flow. Three-dimensional power Doppler image presents early vascular anatomy at the base of the skull with branches evolving laterally to the mesencephalon and cephalic flexure. ${ }^{9}$



Fig. 5: Conventional 3D power Doppler image of fetal brain circulation. (left) Viewfrom the front. Bilateral internal carotid arteries (ICA) and middle cerebral arteries (MCA) and branches of MCA are demonstrated. (right) Oblique view. Anterior cerebral artery (ACA) and pericallosal artery (PcA) are demonstrated 

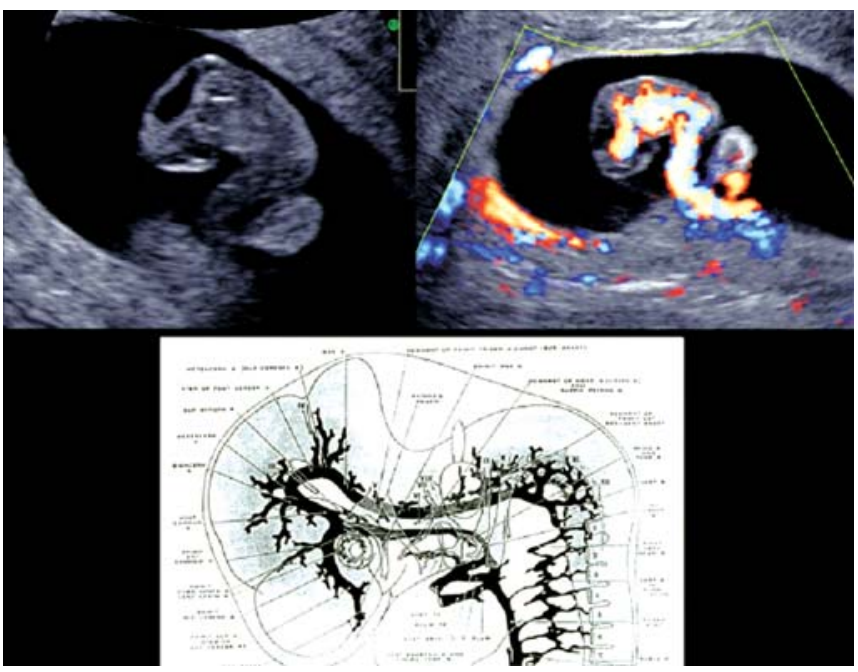

Fig. 6: Early embryonal vascularity at the beginning of 8 weeks Upper left-the sagittal sonographic embryonal image at 7 th week. Upper right-bidirectional power Doppler image on the same cutting section. Lower-the scheme of developing cerebral circulation of embryo in 7th week of gestation with CRL $9 \mathrm{~mm}$ and Carnegie stage 16 (Schematic picture by courtesy of G Centini)


Fig. 7: On the left, coronal 3D power Doppler image of the embryo at 8th week. On the right, the scheme of developing cerebral circulation of embryo in 8th week of gestation with CRL $24 \mathrm{~mm}$ and Carnegie stage 22 (Schematic picture by courtesy of G Centini)

A progressive increase in blood flow velocity is noted for all the cerebral arteries with advance in gestational age. Between the 9th and 10th weeks, diastolic velocity begins to emerge but they are incomplete and inconsistently present. From the 11th week, end-diastolic bloodflow begins to be constant. The posterior lateral choroidal artery derives from the posterior cerebral artery, whereas the lateral choroidal artery derives from the middle cerebral artery and internal carotid artery. Blood flow signals from the choroidal plexus are obtained during 9th and 10th weeks of gestation as subtle color and pulsed Doppler signals at the inner edge of the

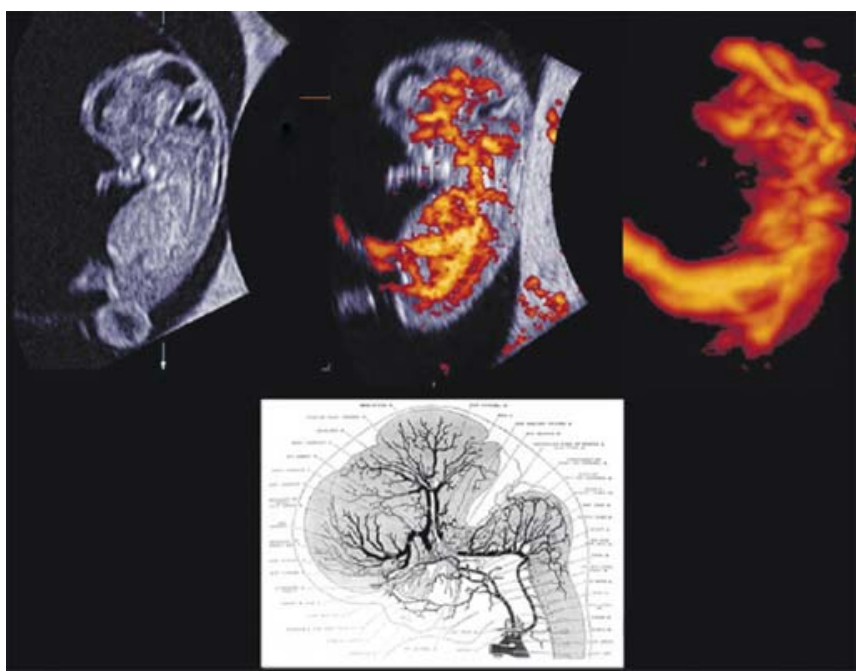

Fig. 8: Early embryonal vascularity at 9th week. Upper left-the sagittal sonographic embryonal image. Upper middle-power Doppler image of fetal vascularity with surface appearance. Upper right-3D power Doppler image of vascular system. Lower-the scheme of developing cerebral circulation of embryo in 8th week of gestation with CRL 24 $\mathrm{mm}$ and Carnegie stage 22 (Schematic picture by courtesy of G Centini)

lateral ventricle choroidal plexus. Visualization of the vessels of the choroid plexus increased and decreased as the gland shrank. This developmental period is also the time of active neurogenesis. Choroid plexus vascularity during 9th and 10th weeks of gestation has two typical features: prominent venous blood flow signals and absence of diastolic flow. ${ }^{2}$ In parallel, the cranial venous system, important for brain drainage develops dramatically. The venous circulation drains the blood into dural sinuses which met in the confluence of the sinuses at the occipital pole, from where the blood flow runs into the jugular veins. As stated before, color/power Doppler and pulsed Doppler enabled to image and evaluate the carotid arteries ${ }^{7}$ and cerebral arterial blood flow. Particularly the middle cerebral arteries (MCA) have been the objective of Doppler measurements and studies as representative blood vessel of the brain which stayed in clinical practice by now. MCA is the largest branch of internal carotid artery supplying approximately $80 \%$ of cerebral hemisphere. In middle and late pregnancy, the greater wings of sphenoid bone, between the anterior and middle fossa are good reference points for locating middle cerebral artery. This vessel consists of four segments: M1, M2, M3 and M4 and that preferable site for Doppler assessment is M1 segment since maintains a more constant diameter. Bilateral common carotid arteries, internal carotid arteries and the basilar arteries were imaged in the coronal plane (Fig. 9, left) and the circle of Willis and MCAs can be demonstrated in the parietal view of 3D power Doppler reconstructed image (Fig. 9, right) at 12th week of gestation. The anterior cerebral artery (ACA) and 

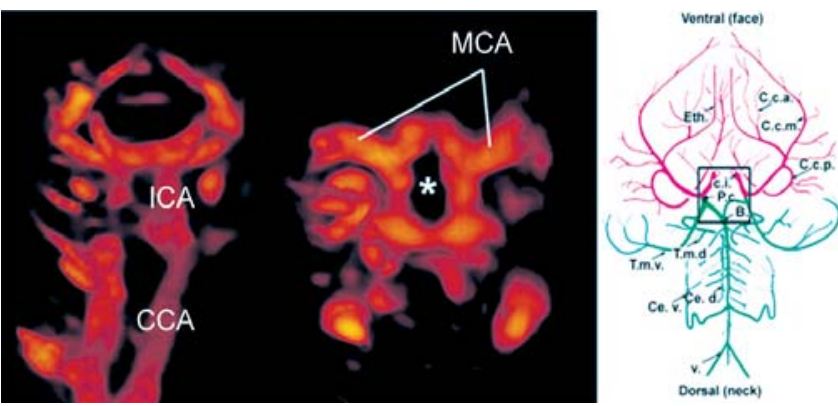

Fig. 9: Vascular structure of normal 12-week-brain by 3D power Doppler. Left side-front back power Doppler image of common carotid arteries (CCA), internal carotid arteries (ICA) and brain basilar arteries. In the middle-3D image from fetal parietal direction. The circle of Willis (asterisk) is clearly visualized. MCA; middle cerebral arteries. On the right-scheme of the circle of Willis (Schematic picture by courtesy of G Centini)

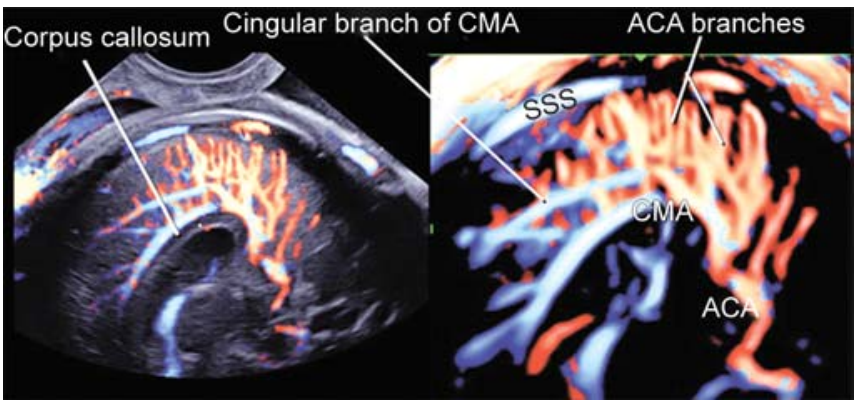

Fig. 10: Anterior cerebral artery and its branches of 24-week normal brain (sagittal view). (left) 2D power Doppler image on the B-mode demonstrates the corpus callosum (CC). (right) 3D reconstructed vascular image. ACA; anterior cerebral artery, CMA; callosomarginal artery, SSS; superior sagittal sinus, Cingular branch of CMA runs along the cingulate gyrus
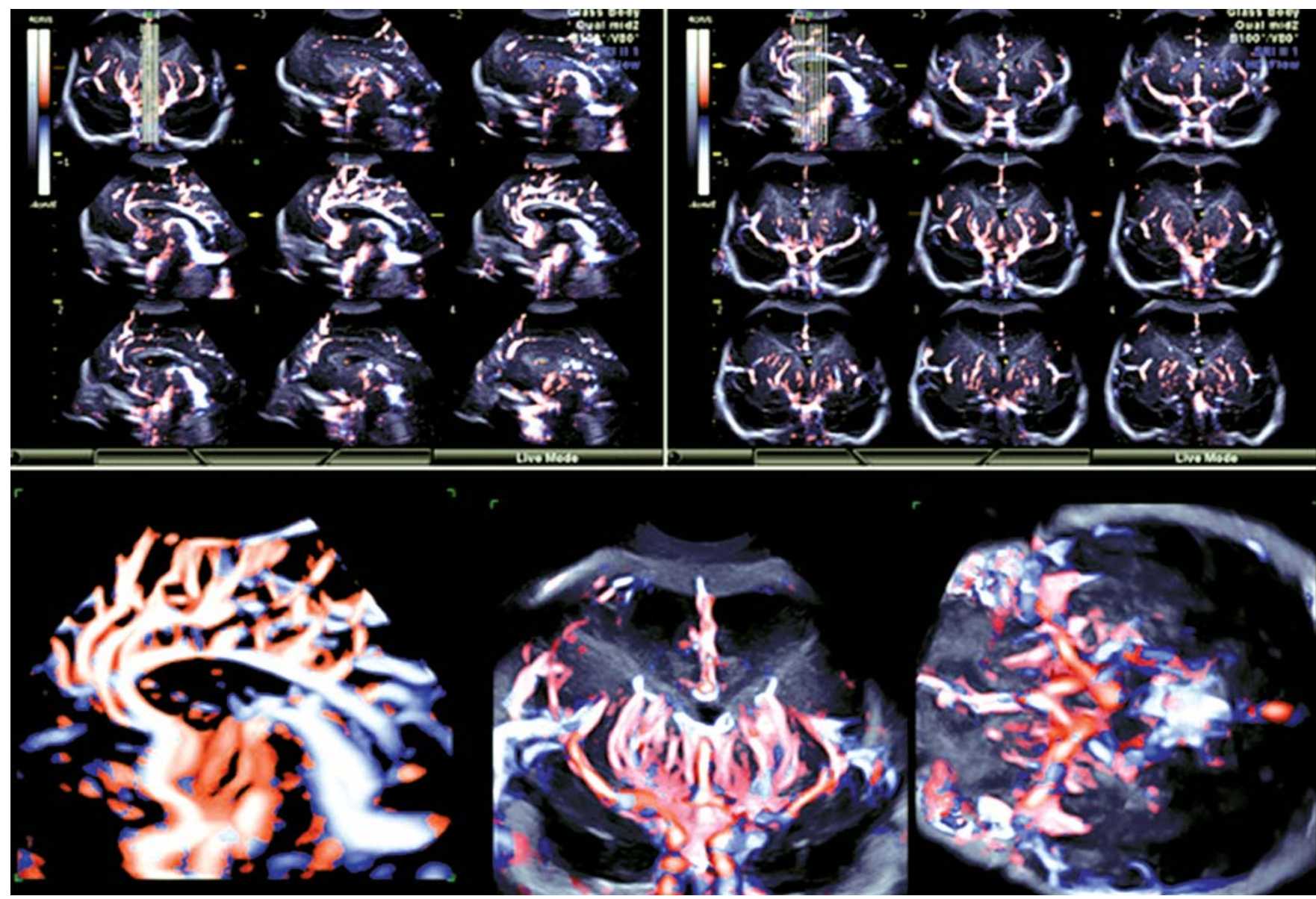

Fig. 11: Tomographic ultrasound imaging and reconstructed 3D angiography of normal cerebral circulation at 31st week. (upper) Tomographic directional power Doppler imaging of sagittal (left upper) and coronal (right upper) sections. Anterior cerebral arteries and their branches are seen on the sagittal plane, middle cerebral arteries and their branches on the coronal plane. (lower) 3D bidirectional power Doppler sonoangiogram of sagittal, coronal and axial sections from left

its branches could be demonstrated in the sagittal plane from the late first trimester. From the late first or early second trimester, MCA, ACA and their branches can be demonstrated by transvaginal 2D/3D power Doppler. In the second trimester, middle cerebral artery is also easy visible and its peak systolic velocity values increases from 22nd to 38th weeks of gestation. ${ }^{12}$ Figure 10 shows the mid-sagittal 2D/3D bidirectional power Doppler images by transvaginal transfontanelle sonography. ACA, callosomarginal artery and their branches are well-demonstrated. Figure 11 shows 
tomographic ultrasound images and reconstructed 3D angiography of normal cerebral circulation at 31 weeks. ACA, MCA, their branches, circle of Willis are clearly depicted. The tomographic ultrasound imaging is useful to obtain an orientation of cerebral vessels. With recent high frequency transvaginal sonography of $12 \mathrm{MHz}$ transducer (Voluson E8, GE Healthcare, USA) has enabled to demonstrated vessels on the cerebral pial surface as shown in Figures 12 and 13. Furthermore, in 2008, Pooh et al succeeded to demonstrate the fine medullary veins running from the cortex towards the subependymal area by bidirectional power Doppler with 3D angiostructural imaging ${ }^{13}$ as shown in Figures 14 to 16. By 3D technology with high-frequency transvaginal sonography, 3D power Doppler image can be acquired and assessed on the orthogonal view. As shown in Figure 14, axial plane (section C) cannot be visualized by 2D transfontanelle sonography and created by 3D computed technology. Conventional 3D power Doppler technology could not depict fine blood vessels, however recent advanced 3D technology provides each tiny blood vessel one by one as shown in right pictures of Figure 14. The medullary veins are detectable from the early second trimesters and develops to numerous showerlike vessels (Fig. 15). Before 20 weeks, medullary veins are demonstrated as red color or red/blue colors by bidirectional power Doppler (Fig. 16, left) and thereafter remarkable rapid development is seen into shower-like

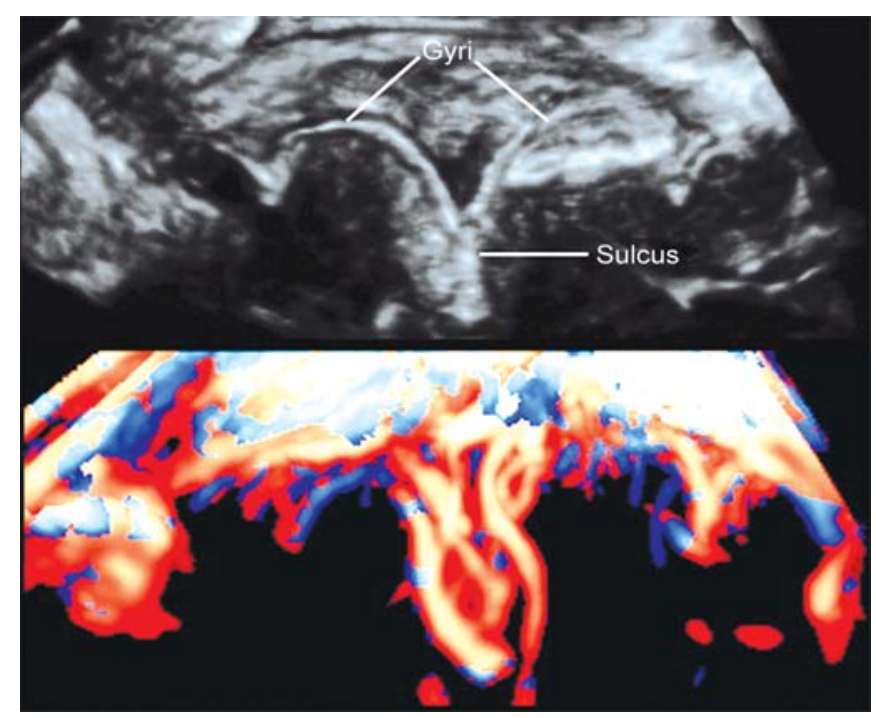

Fig. 12: Coronal view of pial vascularity along cerebral sulci and gyri by 3D bidirectional power Doppler at 33rd week of gestation. (upper) $3 \mathrm{D}$ reconstructed image of the cerebral surface. Protrusion of the brain surface due to gyral formation is clearly demonstrated. (lower) 3D bidirectional power Doppler sonoangiogram of pial vessels along the gyri and sulcus vessels (Fig. 16, right). In the fetal brain, the medullary veins within the deeper cerebral white matter are more developed than the subcortical veins located within the subcortical white matter. ${ }^{14}$ The maldevelopment of medullary veins may indicate brain developmental abnormality, may predict subsequent hydrocephalus, and may predict postnatal neurological deficit. Authors have been investigating assessment of medullary veins in normal and abnormal brain structure and it is expected that the investigation will be one of clues in evaluation of the relations between the fetal brain development and postnatal neurological findings.

\section{VASCULAR ANOMALIES}

Principal pathological causes of spontaneous intracranial hemorrhage in the first year of life are aneurysm, arteriovenous malformations, cavernous vascular malformations and tumors.

After birth, transfontanelle sonography is not always performed for normal babies without any signs or symptoms. 3D subtraction angiography or MR angiography is useful to obtain vascular information postnatally. During fetal period, as it is difficult to use contrast medium or utilize subtraction angiography or MR angiography, 2D/3D color/ power Doppler sonoangiography is a powerful tool for detecting the presence of normal intracranial vessels on the right location, intracranial vascular abnormality and neogenesis of abnormal vascularity.

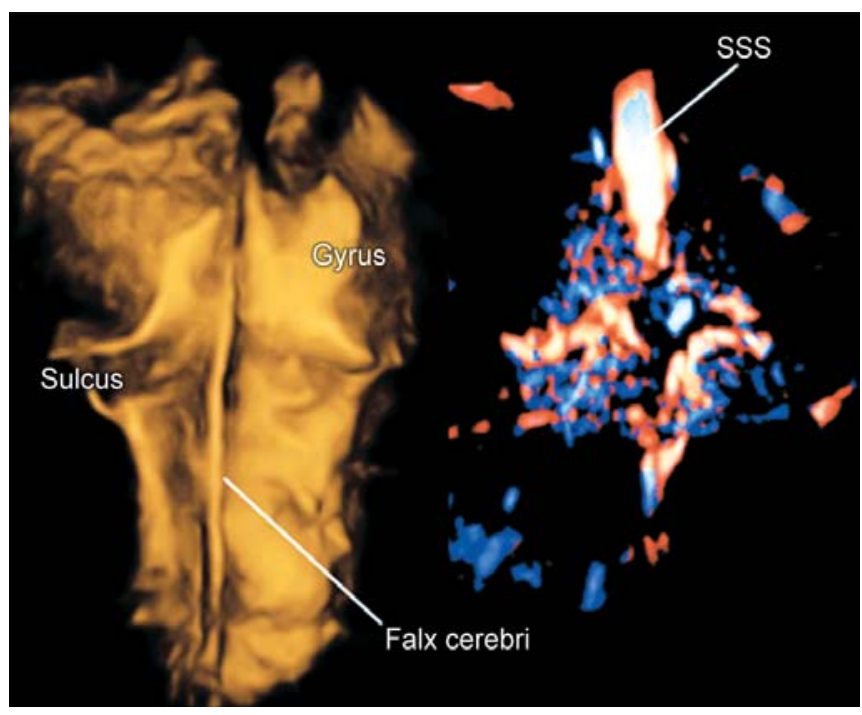

Fig. 13: Parietal view of pial vascularity along cerebral sulci and gyri by 3D bidirectional power Doppler at 30th week of gestation. (left) 3D reconstructed image of the cerebral surface. Falx cerebri and gyral formation is well-demonstrated. (right) 3D bidirectional power Doppler sonoangiogram of superficial vascularity and pial vessels along the gyri and sulcus. SSS; superior sagittal sinus 


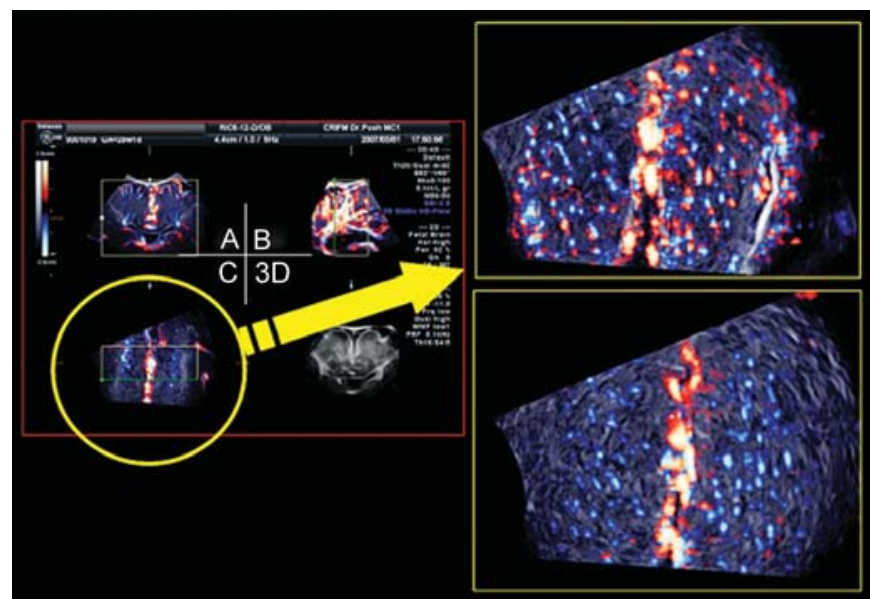

Fig. 14: Advanced 3D technology with high-frequency transvaginal sonography to reconstruct 3D sonoangiogram. (left) Three-orthogonal view of 3D power Doppler of 31st week-normal brain. The image was acquired by transvaginal transfontanelle sonography. Therefore, section A is coronal plane, section B sagittal plane and section $C$ is the axia plane. Axial plane (section $C$ ) is created by 3D technology and cannot be visualized by 2D transfontanelle sonography. Conventional 3D powe Doppler technology could not depict blood vessels, however recent advanced 3D technology provides each fine blood vessel one by one as shown in right figures
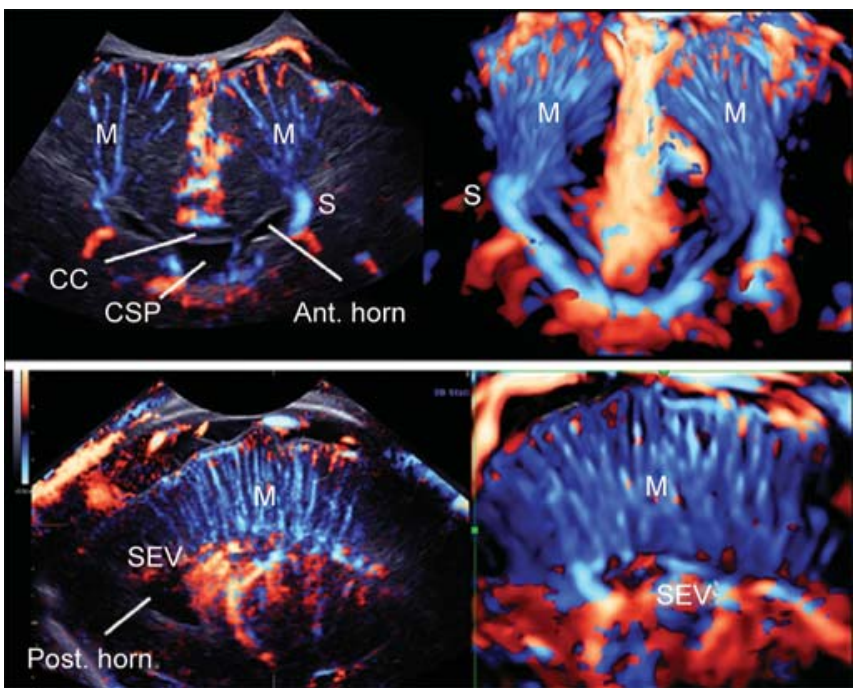

Fig. 15: Normal medullary veins at 28th week. (upper left) Anterior coronal section of 2D bidirectional power Doppler image. Medullary veins (M) and longitudinal caudate vein of Schlesinger (S) are well demonstrated. CC; corpus callosum, CSP; cavum septum pellucidi, Ant.horn; anterior horn of the lateral ventricle. (upper right) Posteroanterior view of 3D reconstructed image of medullary veins. Numerous medullary veins are demonstrated. (lower left) Parasagittal 2D power Doppler image. Cerebral pial vessels are on the surface of cerebrum. Numerous linear vessels run down from the cerebral cortex towards subependymal veins (SEV) are medullary veins (M). (lower) Coronal view of $3 \mathrm{D}$ reconstructed image

\section{VEIN OF GALEN ANEURYSM}

These rare vascular anomalies are a congenital malformation of blood vessels of the brain. The incidence is rare and the

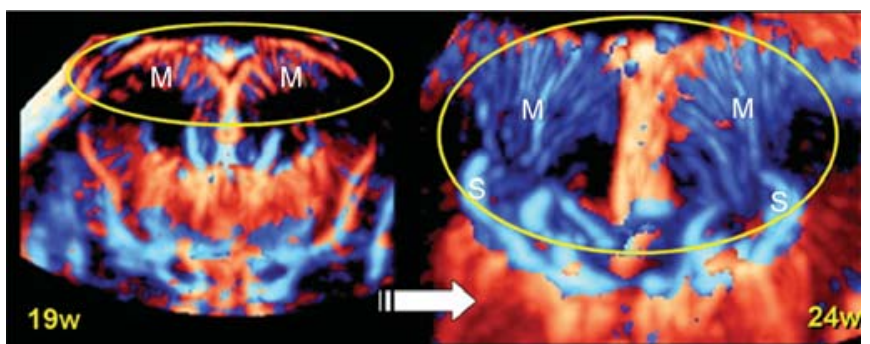

Fig. 16: Development of medullary veins with advance of gestational week. Two images were taken from the same fetus at 19th week of gestation (left) and 24th week (right). At 19th week, medullary veins (M) are premature and most of veins are demonstrated as red color. With advance of gestational weeks, medullary veins (M) develop and became numerous vessels running down from the cerebral cortex towards the longitudinal caudate vein of Schlesinger (S)

etiology and recurrence risk are unknown. The main structure is direct arteriovenous fistulas in which blood shunts from choroidal and/or quadrigeminal arteries into an overlying single median venous sac. These lead to progressive aneurismal dilatation of the vein, whose wall becomes thick and though.

Vein of Galen aneurysm is not real 'aneurysm' but 'arteriovenous malformation (AVM)'. The vein of Galen aneurysmal malformation (VGAM) is a choroidal type of arteriovenous malformation involving the vein of Galen forerunner. This is distinct from an arteriovenous malformation with venous drainage into a dilated, but already formed, vein of Galen. ${ }^{15}$ These anomalies can be associated to anomalies of other systems like cardiomegaly due to high cardiac output, secondary hydrocephalus, macrocrania, cerebral ischemia (intracranial steal phenomenon), and subarachnoid/cerebral/intraventricular hemorrhages. The detection of VGAM includes the visualization of vascular anomaly itself (as shown in Figs 17 and 18). Differential diagnosis includes arachnoid cyst, porencephalic cyst, and intracranial teratoma. Color/power Doppler assessment is easily utilized for differentiation from those other abnormalities. The clinical features differ with the age at presentation. Neonates can have progressive highoutput cardiac failure seen within first few hours after birth. Elder children with this condition may be discovered in the course of investigation of macrocephaly or headaches and subarachnoid hemorrhage in adolescence or adult life.

According to earlier review, outcome did not differ between treated and non-treated group and over $80 \%$ of cases died. ${ }^{16}$ However, recent advances in treatment have improved outcome, such that 60 to $100 \%$ survive and over $60 \%$ have a good neurological outcome. ${ }^{15,17,18}$ The outcome of those diagnosed antenatally and actively treated 
postnatally is better than those diagnosed postnatally due to the opportunity to choose the mode and timing of delivery.

Evaluation of the fetal high-output cardiac state is necessary for the proper obstetrical management. Transfer to a department having all the requisite skills in neonatal intensive care, pediatric interventional radiology, and neonatal anesthesia is required. Percutaneous embolization by microcoils is recent main postnatal treatment and remarkably improved outcome.

\section{VASCULARIZATION OF BRAIN TUMORS}

Brain tumors during fetal and neonatal period is extremely rare. Brain tumors are divided into teratomas and nonteratomatous. Teratomas are most commonly reported and have a variety of their maturity. Nonteratomatous tumors includes neuroepithelial tumor, such as medulloblastoma, astrocytoma, choroids plexus papilloma, choroids plexus carcinoma, ependymoma, ependymoblastoma, and mesenchymal tumor such as craniopharyngioma, sarcoma, fibroma, hemangioblastoma, hemangioma and meningoma, and others of lipoma of the corpus callosum, subependymal giant-cell astrocytoma associated with tuberous sclerosis. ${ }^{19,20}$

Tumor vascularization by prenatal 2D/3D Doppler sonoangiography is shown in Figures 19 to 22. Inside immature teratomas, nonteratomatous malignant tumors and hemangioma, intense vascularization is demonstrated by color/power Doppler due to vascular neogenesis. Recent advances of 3D power Doppler technology provide not only intratumoral vascular structure but also origins of feeding arteries and/or location of draining veins (Fig. 19). The flow waveform of intratumoral vessels has often abnormal pattern of resistance ${ }^{21,22}$ as shown in Figures 19 and 22 and massive neovascularization with low resistant flow may occasionally lead to predicting degree of malignancy. Prenatal information of tumor vascularity is useful to plan postnatal neurosurgical strategy.

\section{CONCLUSION}

Vascularization of the brain is three-dimensional and it is difficult to obtain an orientation of exact location of vessels by conventional Doppler assessment. 2D/3D color/power Doppler ultrasound technology, especially combined with high-frequency transvaginal sonography as described in this chapter is the most preferable modality with noninvasive, and reproducible method. The vascularity information



Fig. 17: 2D/3D power Doppler images of VGAM (Vein of Galen aneurysmal malformation) at 28th week. (Upper left) Mid-sagittal section of 2D power Doppler image. Abnormally dilated vein of Galen is demonstrated. (upper middle) Lateral view of 3D reconstructed power Doppler image. (upper right) Axial section of 2D power Doppler image. Abnormally dilated middle cerebral arteries (MCA) and circle of Willis (W) are demonstrated. Transverse sinus (TS) is also dilated and the blood flow direction is opposite

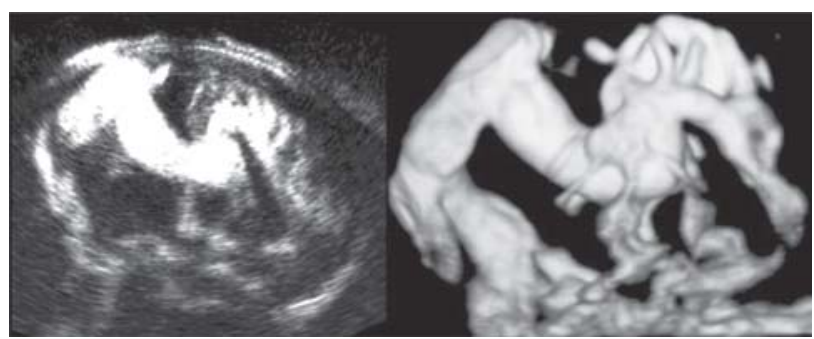

Fig. 18: 2D/3D B-flow images of VGAM (Vein of Galen aneurysmal malformation). (left) Mid-sagittal section of 2D B-flow image. Abnormally dilated vein of Galen is demonstrated. (Right) Lateral view of 3D reconstructed $B$ flow image. This case is VGAM, choroidal type with aplasia of straight sinus. Many of arteries directly run into dilated vein of Galen. VGAM is abnormal arterial-venous shunt

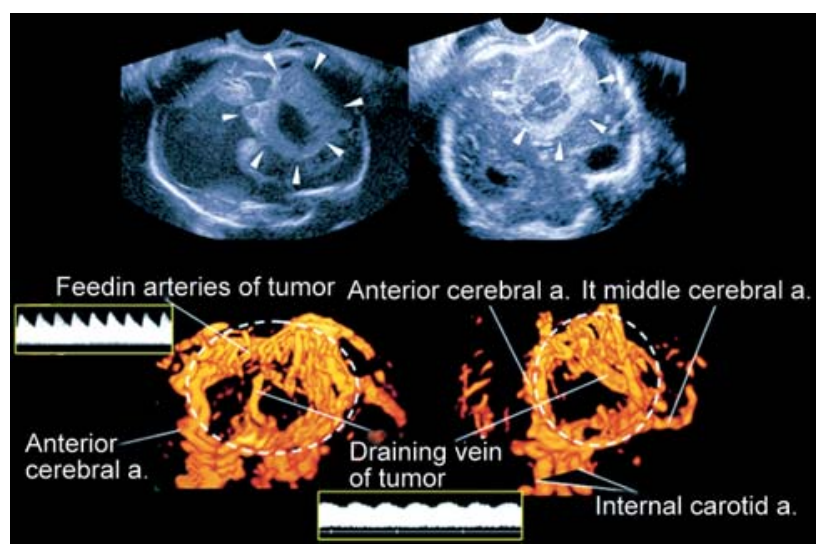

Fig. 19: Ultrasound images and tumoral vascular visualization by 3D power Doppler in a fetus of intracranial tumor with interventricular hemorrhage (35 weeks and 5 days of gestation). (upper) Sagittal and coronal US images. Huge tumor (arrowheads) with hemorrhage within the tumor in the fronto-parietal lobe, complicated with unilateral hydrocephalus with intraventricular hemorrhage. (left lower) Oblique sagittal view of 3D reconstructed power Doppler image from fetal left side. (right lower) Oblique coronal view from fetal frontal side. Tumor is fed by numerous feeding arteries from anterior cerebral artery. Feeder arteries have low resistant flow waveform. One large vein which drains blood from tumor is visible. The draining vein has pulsatile flow 



Fig. 20: Brain tumor at 14th week of gestation. (left) 2D axial section. Huge hyperechogenic mass occupies more than half of cranial cavity. (middle) Fetal MR coronal image. Bilateral hemispheres are oppressed toward parietal portion. (right) Intratumoral vascularity demonstrated by 3D bidirectional power Doppler

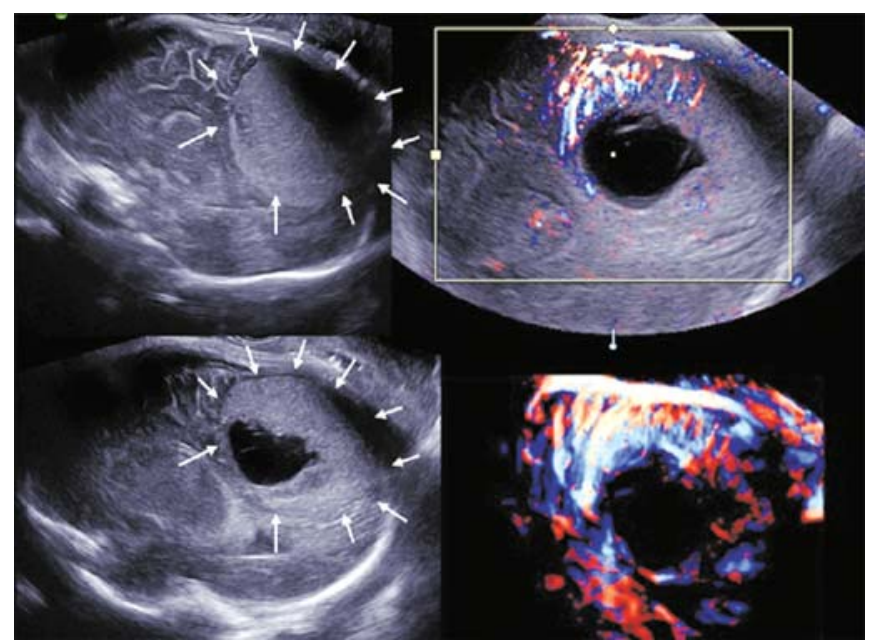

Fig. 21: Brain tumor at 40th week of gestation. (upper left) 2D sagittal section. Echogenic mass (arrows) is demonstrated. (lower left) 2D parasagittal section. Cystic area is depicted inside the mass. 2D power Doppler parasagittal image in the upper right figure and 3D power Doppler reconstructed image in the lower right figure. Numerous intratumoral vascularity is demonstrated

occasionally helps for proper obstetrical and neurological management.

\section{REFERENCES}

1. Kurjak A, Zudenigo D, Predanic M, Kupesic S. Recent advances in the Doppler study of early fetomaternal circulation. J Perinat Med. 1993;21:419-39.

2. Kurjak A, Schulman H, Predanic A, et al. Fetal choroids plexus vascularization assessed by color flow ultrasonography. J Ultrasound Med 1994;13:841-44.

3. Kupesic S, Kurjak A, Babic M. New data on early cerebral circulation. Prenat Neonat Med 1997;2:48-55.

4. Pooh RK, Pooh KH. Transvaginal 3D and Doppler ultrasonography of the fetal brain. Semin Perinat 2001;25:38-43.



Fig. 22: Brain tumor at 24th week of gestation. (upper left) 2D coronal section. Huge mass (arrows) occupied below the hemispheres. Borderline between cerebri and tumor is not clear. (upper right) 2D midsagittal section. (lower left) Three-orthogonal view and reconstructed image of intratumoral vascularity. Chaotic vessels are demonstrated. (lower right) Intratumoral arterial blood flow with low resistance

5. Pooh RK, Pooh KH, Nakagawa Y, Maeda K, Fukui R, Aono T. Transvaginal Doppler assessment of fetal intracranial venous flow. Obstet Gynecol 1999;93:697-701.

6. Risau W. Developing brain produces an angiogenesis factor. Dev Biol 1986;83:3855-59.

7. Virgintino D, Errede M, Robertson D, et al. VEGF expression is developmentally regulated during human brain angiogenesis. Histochem Cell Biol 2003;119(3):227-32.

8. Anne G Osborn. The internal carotid artery: Cavernous, clinoid, ophthalmic and communicating segments In Diagnostic Cerebral Angiography (2nd ed): Lippincott Williams \& Wilkins: Philadelphia 1999;91-93.

9. Kurjak A, Pooh RK. Merce LT, et al. Structural and functional early human development assessed by three-dimensional and four-dimensional sonography. Fertil Steril 2005;84(5):1285-99. 
10. Marsal K, Lingman G, Giles W. Evaluation of the carotid, aortic and umbilical blood velocity waveforms in the human fetus. In 11th Annual Conf Soc Study of Fetal Physiology; 1984:Oxford: Abstract No. C33.

11. Pooh RK, Aono T. Transvaginal power Doppler angiography of the fetal brain. Ultrasound Obstet Gynecol 1996;8(6): 417-21.

12. Gadelha-Costa A, Spara-Gadelha P, Mauad-Filho F, et al. The maximum systolic velocity increases in middle cerebral artery of normal fetus from 22nd to 38th week of gestation. Acta Med Port 2006;19(2):105-08.

13. Pooh RK, Pooh KH. Fetal neuroimiging. Fetal and Maternal Medicine Review 2008;19:1-31.

14. Takashima S, Hirayama A, Okoshi Y, et al. Vascular, axonal and glial pathogenesis of periventricular leukomalacia in fetuses and neonates neuroembryology 2002;1:72-77.

15. Lasjaunias PL, Chng SM, Sachet M, et al. The management of vein of Galen aneurysmal malformations. Neurosurgery 2006;59:184-94.
16. Hoffman HJ, Chuang S, Hendrick EB, et al. Aneurysms of the vein of Galen. Experience at The Hospital for Sick Children, Toronto.J Neurosurg 1982;57:316-22.

17. Campi A, Rodesch G, Scotti G, et al. Aneurysmal malformation of the vein of Galen in three patients: Clinical and radiological follow-up. Neuroradiology 1998;40:816-21.

18. Friedman DM, Verma R, Madrid M, et al. Recent improvement in outcome using transcatheter embolization techniques for neonatal aneurismal malformations of the vein of Galen. Pediatrics 1993;91:583-86.

19. Wakai S, Arai T, Nagai M, et al. Congenital brain tumors. Surg Neurol 1984;21:597-609.

20. Volpe JJ. Brain tumors and vein of Galen malformation. Neurology of the Newborn (4th ed). Philadelphia: WB Saunders 2001;841-56.

21. Pinto V, Meo F, Loiudice L, D’Addario V. Prenatal sonographic imaging of an immature intracranial teratoma. Fetal Diagn Ther 1999;14(4):220-22.

22. Sherer DM, Anyaegbunam A, Onyeije C. Antepartum fetal intracranial hemorrhage, predisposing factors and prenatal sonography: A review. Am J Perinatol 1998;15:431-41. 